\title{
Abacavir/Lamivudine/Zidovudine Maintenance After Standard Induction in Antiretroviral Therapy-Naïve Patients: FREE Randomized Trial Interim Results
}

\author{
Herman G. Sprenger, M.D., Nienke Langebeek, M.Sc., ${ }^{1}$ Paul G.H. Mulder, Ph.D., ${ }^{3}$ \\ Chris H.H. Ten Napel, M.D., Ph.D., ${ }^{4}$ Robert Vriesendorp, M.D., Ph.D., ${ }^{5}$ Andy I.M. Hoepelman, M.D., Ph.D., \\ Jean-Claude Legrand, M.D., ${ }^{7}$ Peter P. Koopmans, M.D., Ph.D., ${ }^{8}$ Marjo E.E. Van Kasteren, M.D., Ph.D., ${ }^{9}$ \\ Bert Bravenboer, M.D., Ph.D., ${ }^{10}$ Reinier W. Ten Kate, M.D., Ph.D., ${ }^{11}$ Paul H.P. Groeneveld, M.D., Ph.D., ${ }^{2}$ \\ Tjip S. van der Werf, M.D., Ph.D.,' Elisabeth H. Gisolf, M.D., Ph.D., ${ }^{2}$ and Clemens Richter, M.D., Ph.D.
}

\begin{abstract}
Maintenance with a triple nucleoside reverse transcriptase Inhibitor (NRTI) regimen after successful induction with a dual NRTI/protease inhibitor (PI) combination may be advantageous, because of low pill burden, favorable lipids, and less drug interactions. This strategy to become free of PI-related problems without losing viral efficacy has not been formally tested. We performed a randomized, open-label, multicenter, 96-week comparative study in antiretroviral therapy (ART)-naïve patients with CD4 $\leq 350$ cells $/ \mathrm{mm}^{3}$ and HIV-1 RNA concentrations (viral load [VL]) greater than 30,000 copies per milliliter. Patients were randomized after reaching VL less than 50 copies per milliliter on two consecutive occasions between 12 and 24 weeks after start of zidovudine/lamuvidine and lopinavir/ritonavir combination. Eligible subjects switched to abacavir/lamivudine/ zidovudine (TZV) or continued the PI-containing regimen. Here we present the 48-week data with virologic success rate (failure: VL $>50$ copies per milliliter). Two hundred seven patients had similar baseline (BL) characteristics: median CD4 180 cells $/ \mathrm{mm}^{3}$, median VL $5.19 \log _{10}$ copies per milliliter. One hundred twenty subjects (58\%) met randomization criteria. Baseline VL differed significantly between dropouts and randomized subjects (median 5.41 versus $5.06 \log _{10}$ copies per milliliter, $p=0.017$ ), as did CD4 cells (median 160 and 200 cells $/ \mathrm{mm}^{3}$, $p=0.044$ ). Sixty-one subjects received TZV and 59 subjects continued NRTIs/PI. At week 48, 2 patients in the TZV group and 5 in the PI group did not have a sustained virologic suppression (log rank test; $p=0.379$ ). CD4 counts increased significantly in both arms. In ART-naïve patients, TZV maintenance had similar antiviral efficacy compared to continued standard ART at 48 weeks after baseline. Patients on successful standard ART can be safely switched to a NRTI-only regimen, at least for the tested time period.
\end{abstract}

\section{Introduction}

C URRENT STANDARD THERAPY OF HIV infection consists of a two-class combination of three antiretroviral (ART) agents $^{1}$ (Panel on Antiretroviral Guidelines for Adult and
Adolescents. Guidelines for the use of antiretroviral agents in HIV-1-infected adults and adolescents. Department of Health and Human Services. December 1, 2009. www.aidsinfo.nih. gov/ContentFiles/AdultandAdolescentGL.pdf). Preferred starting regimens include a backbone of two nucleoside

\footnotetext{
${ }^{1}$ University Medical Center Groningen, Groningen, The Netherlands.

${ }^{2}$ Rijnstate Hospital, Arnhem, The Netherlands.

${ }^{3}$ Erasmus Medical Center, Rotterdam, The Netherlands.

${ }^{4}$ Medical Spectrum Twente, Enschede, The Netherlands.

${ }^{5}$ Medical Center Haaglanden, The Hague, The Netherlands.

${ }^{6}$ University Medical Center Utrecht, Utrecht, The Netherlands.

${ }^{7}$ University Hospital Center, Charleroi, Belgium.

${ }^{8}$ Radboud University Medical Center, Nijmegen, The Netherlands.

${ }^{9}$ Elisabeth Hospital, Tilburg, The Netherlands.

${ }^{10}$ Catharina Hospital, Eindhoven, The Netherlands.

${ }^{11}$ Kennemer Gasthuis, Haarlem, The Netherlands.

${ }^{12}$ Isala Clinics, Zwolle, The Netherlands.
} 
reverse transcriptase inhibitors (NRTIs) either combined with a non-nucleoside reverse transcriptase inhibitor (NNRTI) or a ritonavir-boosted protease inhibitor (PI). In the backbone, one of two NRTIs may be a nucleotide reverse transcriptase inhibitor (NtRTI). These combination antiretroviral therapies (cART) usually suppress HIV replication adequately, with increase of CD4 cell count and at least partial restoration of immunity. Triple-NRTI regimens are generally not recommended, although in ART-naïve patients, the combination of abacavir/lamivudine/zidovudine (TZV) demonstrated virologic activity comparable to indinavir-based, ${ }^{1,2}$ nelfinavirbased, $^{3}$ and atazanavir-based ${ }^{4}$ regimens. In two of these studies $^{1,4}$ virologic response to TZV was suboptimal in subjects with higher baseline viral loads (HIV1-RNA $\geq 100,000$ copies per milliliter). In addition, the AIDS Clinical Trial Group (ACTG) 5095 study showed that TZV was inferior to an efavirenz (EFV)-based regimen regardless of baseline viral load. ${ }^{5}$

Current cART has been associated with significant shortand long-term adverse events, including hyperlipidemia, insulin resistance, and lipodystrophy syndrome. ${ }^{6-8}$ These metabolic side effects appear to translate into an increase of cardiovascular events in HIV-infected patients compared to HIV-seronegative individuals. ${ }^{8-11}$ Because of these and other toxicities, there is a need to explore other therapeutic approaches, including induction-maintenance strategies with simplified treatment regimens.

Simplifying treatment regimens can also improve adherence, which in turn is pivotal to achieve optimal response to treatment. ${ }^{12}$ Complex regimens, higher daily pill burden, and higher dosing frequencies are predictors of nonadherence, with relatively low difference between once or twice daily dosing. ${ }^{13-15}$ PI-based regimens appear associated with lower adherence compared to triple NRTI regimens. ${ }^{16-18}$ Lower adherence to PI-based cART than to NNRTI-based regimens has also been shown. ${ }^{19,20}$ Similar adherence was observed during maintenance phase between patients on a NRTI-only regimen or a NNRTI-based regimen. ${ }^{21}$ Compared to continued quadruple NNRTI-based treatment, adherence to triple NRTI maintenance therapy was better. ${ }^{22}$

Earlier studies addressing a switch to triple NRTI included patients who had not been ART-naïve from the start, resulting in suboptimal responses. ${ }^{17,21,23-27}$ Subsequent studies exploring the induction-maintenance concept in ART-naïve patients showed similar virologic success (i.e., noninferiority) for triple NRTI regimens compared to two-class cART. ${ }^{16,21,22,28-31}$ However, some of these studies were not entirely prospective, ${ }^{16,21,29}$ or did not have a comparative design for the maintenance phase, ${ }^{28,30}$ or used poorly tolerated quadruple regimens as induction regimens. ${ }^{22,32}$

Here we describe a randomized, prospective study in ARTnaïve patients using a standard triple cART as induction therapy, followed by maintenance with TZV in those who reached an undetectable plasma viral load (less than 50 copies per milliliter). We report the interim results at 48 weeks after baseline, specifically addressing the question of virologic efficacy of maintenance with single-class ART.

\section{Methods}

\section{Study population}

HIV-1-infected, ART-naïve adults were eligible with a CD4 cell count $\leq 350$ cells $/ \mathrm{mm}^{3}$ and an HIV-1 RNA level 30,000 copies per milliliter or more at screening. Exclusion criteria included diabetes mellitus or being treated for abnormality of the lipid spectrum. Laboratory exclusion criteria were hemoglobin $<10 \mathrm{~g} / \mathrm{dL}$ (male) or $9 \mathrm{~g} / \mathrm{dL}$ (female), absolute neutrophil count $<1000$ cells $/ \mathrm{mm}^{3}$, platelet count $<75,000$ cells $/ \mathrm{mm}^{3}$, transaminases more than 5 times the upper limit of normal (ULN), total bilirubin $>2 \mathrm{mg} / \mathrm{L}$, serum pancreatic amylase more than 1.5 times the ULN, fasting glucose $>6.9 \mathrm{mmol} / \mathrm{L}$ or nonfasting glucose $>11 \mathrm{mmol} / \mathrm{L}$, fasting triglyceride level $>4 \mathrm{mmol} / \mathrm{L}$, or fasting LDL cholesterol $>5 \mathrm{mmol} / \mathrm{L}$ or low-density lipoprotein/high-density lipoprotein (LDL/HDL) ratio $>5$. Patients were also excluded if they had a history of cardiovascular events or diabetes mellitus, combined with fasting triglyceride level $>3 \mathrm{mmol} / \mathrm{L}$, or fasting LDL cholesterol $>4 \mathrm{mmol} / \mathrm{L}$, or LDL/HDL ratio $>4$.

\section{Study design and study sites}

FREE is an investigator-initiated, randomized, open-label, 96-week study conducted at 10 sites in the Netherlands and 1 site in Belgium. During the induction phase, all patients were treated with 1 fixed-dose tablet of lamivudine (3TC) $150 \mathrm{mg} /$ zidovudine (ZDV) $300 \mathrm{mg}$ (Combivir ${ }^{\circledR}$; GlaxoSmtihKline, Zeist, The Netherlands, and Genval, Belgium) twice daily and with 3 capsules lopinavir (LPV) $133 \mathrm{mg} /$ ritonavir (r) $33 \mathrm{mg}$ (Kaletra ${ }^{\circledR}$; Abott, Hoofddorp, The Netherlands and LouvainLa-Neuve, Belgium) twice daily. In 2005, the manufacturer changed the formulation of LPV/r, and the capsules were replaced by tablets $\times 200 / 50 \mathrm{mg}$, 2 tablets taken twice daily.

Only patients with HIV-1 RNA levels less than 50 copies per milliliter between week 12 and week 24, measured on two consecutive visits at least 4 weeks apart, were eligible for randomization into the maintenance phase. Patients were randomized on a 1:1 basis by computer-generated allocation to either continue 3TC/ZDV and LPV/r (PI arm) or to 1 fixeddose tablet of Abacavir $300 \mathrm{mg} / 3 \mathrm{TC} 150 \mathrm{mg} / \mathrm{ZDV} 300 \mathrm{mg}$ (Trizivir; GlaxoSmtihKline, Zeist, The Netherlands, and Louvain-La-Neuve, Belgium; the TZV arm), twice daily. A minimization rule was applied for the patient factor: HIV-1 RNA copy number. Patients were stratified in three groups: $30,000-100,000$; more than 100,000-1,000,000; or more than $1,000,000$ copies per milliliter at study entry. Screening evaluation included a clinical assessment and laboratory evaluations: plasma HIV-1 RNA, CD4 cell count, hematology, clinical chemistries, and collections of a plasma sample for retrospective research for viral resistance mutations. Screening for the presence of the human leukocyte antigen (HLA)-B*5701 subtype was not performed. In recent years a statistically significant correlation with the abacavir hypersensitivity reaction and the presence of this allele has been shown. ${ }^{33}$ At the time this study was started routine genotyping for the HLA-B ${ }^{* 5701}$ allele was not available. On-study evaluations included clinic visits at baseline and at week 4,8 , 12,18 , and 24, for the induction phase; and at weeks 36, 48, 60, 72,84 , and 96 for the maintenance phase. HIV-1 RNA was assessed locally at each visit using the Roche Ultrasensitive Assay (Roche Diagnostics, Indianapolis, IN) with a lower limit of detection of less than 50 copies per milliliter.

\section{End points}

The primary end point of the study was antiviral efficacy at week 96, defined as plasma HIV-1 RNA less than 400 copies 
per milliliter. The purpose of the study was to assess treatment equivalence of this endpoint between both groups. Secondary end points included safety and tolerability; effect on absolute and cumulative $\mathrm{CD} 4^{+}$cell count changes compared to baseline; use of cART related comedication, especially lipid-lowering agents; and time to treatment failure (HIV-1 RNA less than 50 copies per milliliter). Here we addressed the secondary end points at week 48: virologic efficacy (sustained viral load suppression less than 50 copies per milliliter), and premature discontinuation of allocated ART for any reason. We considered detectable viral load as well as treatment discontinuation as treatment failure for the present interim analysis.

\section{Sample size}

With an expected antiviral efficacy percentage of $80 \%$ at week 96 and an equivalence limit of 20 percent points (as assumed in the protocol), 50 randomized evaluable subjects per group are needed to show equivalence with $80 \%$ power, using a test size of 0.05 (one-sided).

\section{Statistical analyses}

Time to virologic failure was analyzed using the KaplanMeier method, and the log-rank test was used to compare survivor functions (sustaining HIV-RNA less than 50 copies per milliliter) between groups. The $95 \%$ confidence interval was estimated for the difference in the percentages of treatment failure (virologic failure or premature discontinuation) between the two groups. All tests for secondary end points were two-sided with a confidence level of $95 \%$. Changes in the absolute numbers of CD4 cells during the randomization phase were analyzed using mixed-model analysis of variance (ANOVA). Statistical analyses were performed using SPSS version 16 (SPSS, Inc., Chicago, IL).

\section{Ethics}

The protocol was approved by Institutional Review Boards of all hospitals involved. All participants gave consent after written information had been given, in accordance with the national and international legislation, as well as the Declaration of Helsinki.

\section{Role of sponsor}

This was an investigator-driven study; the sponsor was not involved in the design, data analysis, draft of the paper, or in the decision to submit the paper for publication.

The protocol (with revisions and updates) was registered at Clinicaltrials.com; Registration Identifier Number: NCT00405925.

\section{Results}

\section{Patient disposition}

Of 241 patients screened, 207 were enrolled in the induction phase. Table 1 shows their demographic and baseline characteristics. Patients at baseline were predominantly male, with a median age of 40.8 years (range, 19.4-78.1), had a median baseline HIV-1 RNA $5.19 \log _{10}$ copies per milliliter (range, 2.97-7.45), and a median CD4 cell count $180 / \mathrm{mm}^{3}$ (range, 10-440). Eighty-seven patients (36\%) did not reach the maintenance phase (Fig. 1) primarily because they did not achieve virologic suppression (HIV-1 RNA less than 50 copies per milliliter on two occasions) before week 24 (47 patients, $54 \%$ ), or because of side effects (22 patients, $25 \%$ ). Of baseline variables only HIV-1 RNA level and CD4 cells differed significantly between the patients who did not reach the maintenance phase of the study (drop outs; HIV-1 RNA median $5.41 \log _{10}$ copies per milliliter) and the patients who could be randomized (median $5.06 \log _{10}$ copies per milliliter). Median CD4 cell count was 160 cells $/ \mathrm{mm}^{3}$ in the dropouts and 200 cells $/ \mathrm{mm}^{3}$ in the randomized group. A total of 120 patients were randomized to either continuation of the induction regimen $(n=59)$ or to switch to TZV $(n=61)$. The two groups were similar, and characteristics did not change between initial enrolment of participants and at randomization (Table 1). Nine patients were randomized, but did not start with the maintenance phase, 5 patients randomized into the PI arm, and 4 into the TZV arm. These 9 patients had no follow-up visits during the maintenance phase and were excluded from the analyses. Figure 1 provides details about these study participants that did not follow the allocated treatment regimen.

Table 1. Demographic and Baseline Characteristics of Patients

\begin{tabular}{|c|c|c|c|}
\hline & \multirow{2}{*}{$\begin{array}{c}\text { Induction phase } \\
\begin{array}{c}C B V / L P V / r \\
(\mathrm{n}=207)\end{array}\end{array}$} & \multicolumn{2}{|c|}{ Maintenance phase } \\
\hline & & $\begin{array}{l}C B V / L P V / r \\
(\mathrm{n}=59)\end{array}$ & $\begin{array}{c}\mathrm{TZV} \\
(\mathrm{n}=61)\end{array}$ \\
\hline Median age (range), yr & $39.7(19.4-78.1)$ & $40.3(24.7-62.0)$ & $43.1(21.9-68.6)$ \\
\hline Male sex, $n(\%)$ & $181(87 \%)$ & $52(88 \%)$ & $50(82 \%)$ \\
\hline \multicolumn{4}{|l|}{ Median baseline plasma HIV-1 RNA } \\
\hline Level (range), $\log _{10}$ copies $/ \mathrm{mL}$ & $5.19(2.97-7.45)$ & $5.03(4.17-6.61)$ & $5.07(2.97-6.18)$ \\
\hline$\geq 100,000$ copies $/ \mathrm{mL}$ & $115(60 \%)$ & $29(49 \%)$ & $33(54 \%)$ \\
\hline \multicolumn{4}{|l|}{$\overline{\text { Median }}$ CD4 cell count at baseline } \\
\hline cells $/ \mathrm{mm}^{3}$ (range) & $180(10-440)$ & $207(10-370)$ & $195(10-437)$ \\
\hline$<50$ cells $/ \mathrm{mm}^{3}, n(\%)$ & $31(15 \%)$ & $6(11 \%)$ & $9(16 \%)$ \\
\hline$>50-<200$ cells $/ \mathrm{mm}^{3}, n(\%)$ & $73(35 \%)$ & $18(34 \%)$ & $20(35 \%)$ \\
\hline $\begin{array}{l}\text { Median CD } 4 \text { cell count at randomization } \\
\text { cells } / \mathrm{mm}^{3} \text { (range) }\end{array}$ & NA & $350(50-610)$ & $310(50-780)$ \\
\hline
\end{tabular}

NA, not applicable. 


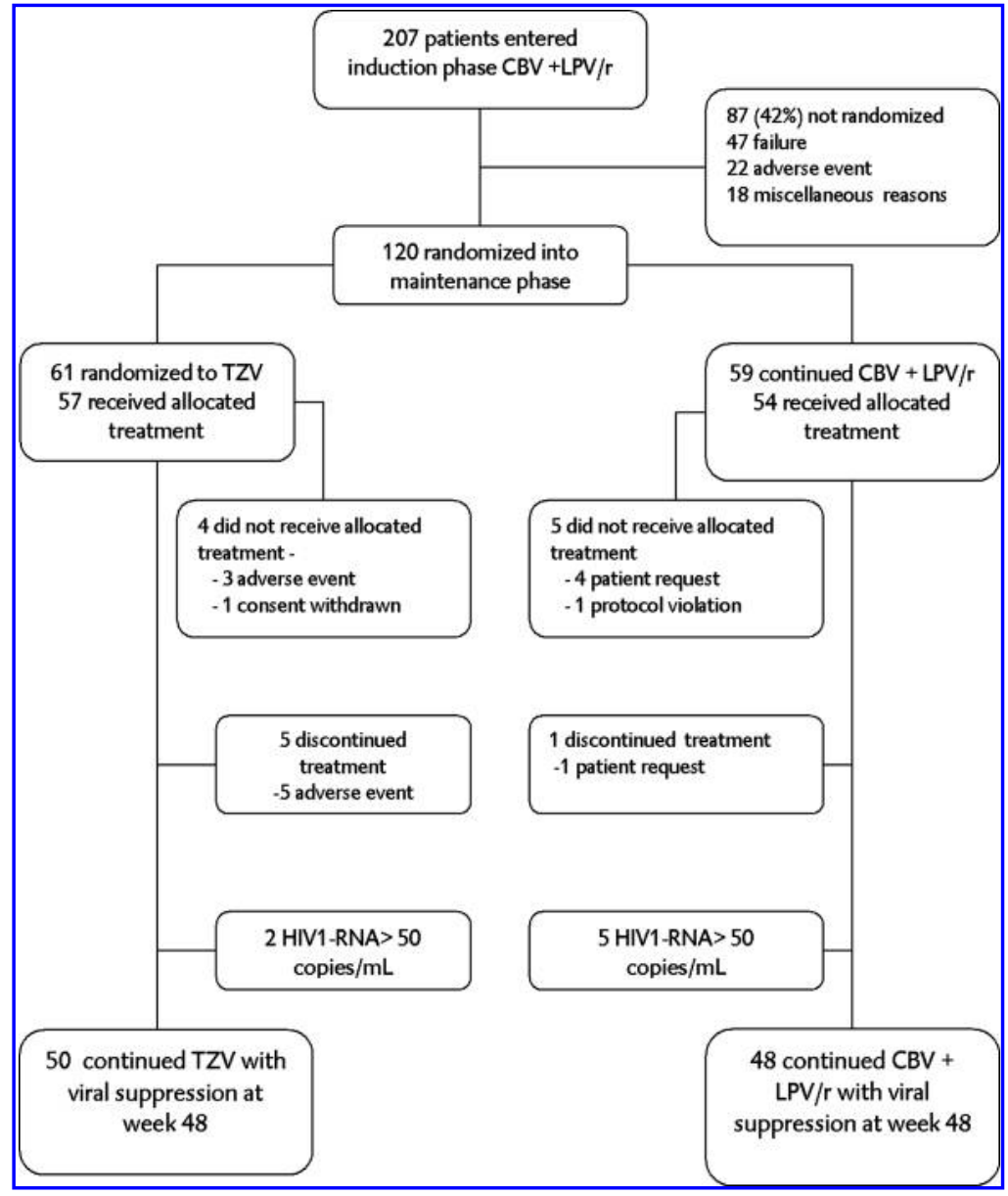

FIG. 1. Flow chart and disposition of study participants in the FREE trial; interim results at week 48 .

\section{Virologic response and treatment (dis-)continuation}

At week 48, the proportion of patients with treatment failure (virologic or treatment discontinuation) was $12 \%(7 / 57)$ in the TZV group and $11 \%(6 / 54)$ in the PI group. The difference between the TZV group and the PI group was 1.2 percentage points (TZV-PI; 95\% CI-11.0 to +13.4 ). With these confidence limits lying wholly in the predefined range equivalence is demonstrated. In the TZV group most failures $(5 / 7)$ were not virologic but premature discontinuations because of adverse events. Five patients in the PI group had HIV-1 RNA 50 copies per milliliter or more at any one time before the end of week 48 , compared to two patients in the TZV group (log rank test; $p=0.379$ ). In two of the patients in the PI group only a transient viral "blip" (defined as a single isolated or recurrent but transient episode of detectable low-level viremia greater than 50 copies per milliliter) was detected (at week 24 and at week 36), the three other patients had HIV-RNA greater than 50 copies per milliliter for the first time at their visit in week 48 . In the TZV group one patient had HIV-1 RNA greater than 50 copies per milliliter in week 36 , with no result known at week 48. The second patient in the TZV group had HIV-1 RNA greater than 50 copies per milliliter only at week 48 .

There was an increase of CD4 cells in both maintenance treatment arms at week 48 compared to their CD4 cell counts at randomization: in the PI group 95 (95\% CI 42-149) CD4 cells $/ \mathrm{mm}^{3}(p=0.001)$, and in the TZV group 69 (95\% CI 25113) CD4 cells $/ \mathrm{mm}^{3}(p=0.002)$. The increase of CD4 cells at week 48 was not significantly different between groups $(p=0.46)$.

\section{Discussion}

The FREE trial is the first study exploring a $100 \%$ prospective, well-designed induction-maintenance strategy in ART-naïve patients after induction therapy with standard cART. We show that viral suppression can be maintained by a single-class regimen after successful induction by two-class triple cART standard induction therapy. At week 48, virologic success rates with the triple NRTI regimen (TZV: $96.5 \%$ ) and the continued standard PI-based cART regimen (90.7\%) were similar.

Our interim results confirm findings from two earlier studies showing that the induction-maintenance approach is effective ${ }^{22,32}$ but induction therapy in these studies consisted of four drugs, TZV, and EFV. In these studies the four-drug regimens had high discontinuation rates (24\% and $37 \%)$. The Trizefal study ${ }^{30}$ compared two quadruple induction regimens, TZV with either LPV/r or EFV, which after successful induction were followed by maintenance therapy with TZV. In the induction phase there was also a high discontinuation rate: $45 \%$ did not reach the maintenance phase primarily because of adverse events. Although we show that a triple cART as induction therapy can be followed successfully by a singleclass maintenance regimen, our dropout was fairly high, mainly because of strict inclusion criteria for the decision to 
switch to maintenance. Undetectable viral load (HIV-1 RNA $<50$ copies per milliliter) on two different visits 4-6 weeks apart within 24 weeks after baseline was an unattainable goal for nearly half the patients; only $58 \%$ of patients enrolled at baseline could therefore be randomized. Patients (42\%) who could not be randomized had significantly higher HIV-1 RNA copies per milliliter, and a lower CD4 count at baseline. Other studies used longer induction periods (24-48 weeks $)^{22,30}$ and such policy would have increased the sample size of our study subjects. The rationale to determine a relatively short induction period was the fear to include subjects with pre-existing resistant mutant (quasi-)species virus with an enhanced chance to fail on a single class NRTI maintenance regimen. Since the average second phase HIV-1 RNA decay after cART initiation is $2-4$ weeks on average, ${ }^{34}$ treatment of approximately 6-12 half-lives, or approximately $24-48$ weeks, would result in a substantially reduced residual viral burden at the time of treatment simplification. Our findings at 48 weeks suggest that such a policy to prolong the induction phase to reduce the risk for failure may not be necessary for those subjects that reach undetectable viral loads at week 20 and week 24 , as virologic failure was equally uncommon in both arms of our study analyzed at week 48 .

Potential weaknesses of our study are first, the limited sample size; had the design allowed for randomization with viral suppression after week 24 , this would have increased the sample size. Second, lack of concealment may have influenced failure rates, especially because the major source of failure was not virologic failure per se, but rather stopping study medication "for any reason." Third, at this interim analysis at week 48 , it is too early to predict the success of simplified treatment with TZV if such maintenance regimen were continued for longer periods of time.

The simplification of cART after a successful induction period to a single-class regimen may be advantageous, even if such simplified treatment were only justified for limited periods of time. Triple NRTI regimens offer convenient dosing regimens with a very low pill burden (two pills per day) leading to better adherence, show favourable lipid profiles, $^{16,22,25}$ and result in fewer potentially serious drug interactions than standard cART. This may be helpful if during intercurrent medical or surgical events, drug-drug interactions need to be avoided, e.g., the use of rifamycin-based regimens for tuberculosis and non tuberculous mycobacterial infections; anti-convulsive, anti-arrhythmic, or antimalaria therapy. Based on our observations, patients with viral suppression who need to interrupt PI or NNRTI agents, can be safely managed with single class NRTI - at least temporarilywith acceptably low chances of viral failure. With continued viral suppression less than 50 copies per milliliter, the chances of acquiring drug resistance are generally low. ${ }^{35}$

Although there was a low virologic failure rate after randomization in both arms in our study at week 48 , results at week 96 have to be awaited to determine the safety and efficacy of single-class NRTI maintenance therapy during longer periods of time.

\section{Acknowledgments}

Presented in part at the Ninth International Congress on Drug Therapy in HIV Infection, November 9-13, 2008, Glasgow, UK.
Supported by an unrestricted grant from GlaxoSmithKline.

\section{Author Disclosure Statement}

No competing financial interests exist.

\section{References}

1. Staszewski S, Keiser P, Montaner J, et al. Abacavir-lamivudinezidovudine vs indinavir-lamivudine-zidovudine in antiretroviralnaive HIV-infected adults: A randomized equivalence trial. JAMA 2001;285:1155-1163.

2. Vibhagool A, Cahn P, Schechter M, et al. Triple nucleoside treatment with abacavir plus the lamivudine/zidovudine combination tablet (COM) compared to indinavir/COM in antiretroviral therapy-naive adults: Results of a 48-week open-label, equivalence trial (CNA3014). Curr Med Res Opin 2004;20:1103-1114.

3. Kumar PN, Rodriguez-French A, Thompson MA, et al. A prospective, 96-week study of the impact of Trizivir, Combivir/nelfinavir, and lamivudine/stavudine/nelfinavir on lipids, metabolic parameters and efficacy in antiretroviralnaive patients: Effect of sex and ethnicity. HIV Med 2006; 7:85-98.

4. Kumar PN, Salvato P, Lamarca A, et al. A randomized, controlled trial of initial anti-retroviral therapy with abacavir/lamivudine/zidovudine twice-daily compared to atazanavir once-daily with lamivudine/zidovudine twice-daily in HIV-infected patients over 48 weeks (ESS100327, the ACTION Study). AIDS Res Ther 2009;6:3.

5. Gulick RM, Lalezari J, Goodrich J, et al. Maraviroc for previously treated patients with R5 HIV-1 infection. N Engl J Med 2008;359:1429-1441.

6. Galli M, Ridolfo AL, Adorni F, et al. Body habitus changes and metabolic alterations in protease inhibitor-naive HIV-1infected patients treated with two nucleoside reverse transcriptase inhibitors. J Acquir Immune Defic Syndr 2002; 29:21-31.

7. Vergis EN, Paterson DL, Wagener MM, et al. Dyslipidaemia in HIV-infected patients: Association with adherence to potent antiretroviral therapy. Int J STD AIDS 2001;12:463468.

8. Walli RK, Michl GM, Bogner JR, et al. Improvement of HAART-associated insulin resistance and dyslipidemia after replacement of protease inhibitors with abacavir. Eur J Med Res 2001;6:413-421.

9. Henry K, Melroe H, Huebsch J, et al. Severe premature coronary artery disease with protease inhibitors. Lancet 1998;351:1328.

10. Currier JS, Taylor A, Boyd F, et al. Coronary heart disease in HIV-infected individuals. I Acquir Immune Defic Syndr 2003;33:506-512.

11. Friis-Moller N, Reiss P, Sabin CA, et al. Class of antiretroviral drugs and the risk of myocardial infarction. $\underline{N}$ Engl J Med 2007;356:1723-1735.

12. Paterson DL, Swindells S, Mohr J, et al. Adherence to protease inhibitor therapy and outcomes in patients with HIV infection. Ann Intern Med 2000;133:21-30.

13. Glass TR, De Geest S, Weber R, et al. Correlates of selfreported nonadherence to antiretroviral therapy in HIVinfected patients: The Swiss HIV Cohort Study. I Acquir Immune Defic Syndr 2006;41:385-392.

14. Protopopescu C, Raffi F, Roux P, et al. Factors associated with non-adherence to long-term highly active antiretroviral therapy: A 10 year follow-up analysis with correction for the 
bias induced by missing data. I Antimicrob Chemother 2009;64:599-606.

15. Atkinson MJ, Petrozzino JJ. An evidence-based review of treatment-related determinants of patients' nonadherence to HIV medications. AIDS Patient Care STDs 2009;23:903-914.

16. Clumeck N, Goebel F, Rozenbaum W, et al. Simplification with abacavir-based triple nucleoside therapy versus continued protease inhibitor-based highly active antiretroviral therapy in HIV-1-infected patients with undetectable plasma HIV-1 RNA. AIDS 2001;15:1517-1526.

17. Opravil M, Hirschel B, Lazzarin A, et al. A randomized trial of simplified maintenance therapy with abacavir, lamivudine, and zidovudine in human immunodeficiency virus infection. J Infect Dis 2002;185:1251-1260.

18. Cahn P, Vibhagool A, Schechter M, et al. Predictors of adherence and virologic outcome in HIV-infected patients treated with abacavir- or indinavir-based triple combination HAART also containing lamivudine/zidovudine. Curr Med Res Opin 2004;20:1115-1123.

19. Trotta MP, Ammassari A, Cozzi-Lepri A, et al. Adherence to highly active antiretroviral therapy is better in patients receiving non-nucleoside reverse transcriptase inhibitorcontaining regimens than in those receiving protease inhibitor-containing regimens. AIDS 2003;17:1099-1102.

20. Lazo M, Gange SJ, Wilson TE, et al. Patterns and predictors of changes in adherence to highly active antiretroviral therapy: Longitudinal study of men and women. $\underline{\text { Clin Infect }}$ Dis 2007;45:1377-1385.

21. Bonjoch A, Paredes R, Galvez J, et al. Antiretroviral treatment simplification with 3 NRTIs or 2 NRTIs plus nevirapine in HIV-1-infected patients treated with successful first-line HAART. J Acquir Immune Defic Syndr 2005;39:313-316.

22. Markowitz M, Hill-Zabala C, Lang, J et al. Induction with abacavir/lamivudine/zidovudine plus efavirenz for 48 weeks followed by 48-week maintenance with abacavir/lamivudine/ zidovudine alone in antiretroviral-naive HIV-1-infected patients. J Acquir Immune Defic Syndr 2005;39:257-264.

23. Abgrall S, Yeni PG, Bouchaud O, et al. Switch from a first virologically effective protease inhibitor-containing regimen to a regimen containing efavirenz, nevirapine or abacavir. AIDS 2006;20:2099-2106.

24. Katlama C, Fenske S, Gazzard B, et al. TRIZAL study: Switching from successful HAART to Trizivir (abacavirlamivudine-zidovudine combination tablet): 48 weeks efficacy, safety and adherence results. HIV Med 2003;4: 79-86.

25. Keiser PH, Sension MG, DeJesus E, et al. Substituting abacavir for hyperlipidemia-associated protease inhibitors in HAART regimens improves fasting lipid profiles, maintains virologic suppression, and simplifies treatment. BMC Infect Dis 2005;5:2.
26. Maggiolo F, Ripamonti D, Ravasio L, et al. Outcome of 2 simplification strategies for the treatment of human immunodeficiency virus type 1 infection. Clin Infect Dis 2003; 37:41-49.

27. Wolbers M, Opravil M, von Wyl V, et al. Predictors of optimal viral suppression in patients switched to abacavir, lamivudine, and zidovudine: the Swiss HIV Cohort Study. AIDS 2007;21:2201-2207.

28. de Truchis P, Mathez D, Force G, et al. Long-term control of viral residual replication under maintenance therapy with Trizivir after a quadruple induction regimen in HIV-1infected adults (Suburbs trial). HIV Clin Trials 2007;8:102-104.

29. John M, McKinnon EJ, James IR, et al. Randomized, controlled, 48-week study of switching stavudine and/or protease inhibitors to combivir/abacavir to prevent or reverse lipoatrophy in HIV-infected patients. J Acquir Immune Defic Syndr 2003;33:29-33.

30. Mallolas J, Pich J, Penaranda M, et al. Induction therapy with trizivir plus efavirenz or lopinavir/ritonavir followed by trizivir alone in naive HIV-1-infected adults. AIDS 2008;22: 377-384.

31. Moyle GJ, Baldwin C, Langroudi B, et al. A 48-week, randomized, open-label comparison of three abacavir-based substitution approaches in the management of dyslipidemia and peripheral lipoatrophy. J Acquir Immune Defic Syndr 2003;33:22-28.

32. de Wit S, Johnson M, Gazzard B, et al. Randomised comparison of maintenance with Trizivir $[\mathrm{TZV}]+$ efavirenz [EFV]: TIME study [Abstract No.PL2.4]. 7th Interantional Congress on Drug Therapy in HIV Infection.Glasgow, Scotland: November 14-18, 2004.

33. Mallal S, Phillips E, Carosi G, et al. HLA-B*5701 screening for hypersensitivity to abacavir. N Engl J Med 2008;358:568579.

34. Perelson AS, Essunger P, Cao Y, et al. Decay characteristics of HIV-1-infected compartments during combination therapy. Nature 1997;387:188-191.

35. Kieffer TL, Finucane MM, Nettles RE, et al. Genotypic analysis of HIV-1 drug resistance at the limit of detection: Virus production without evolution in treated adults with undetectable HIV loads. J Infect Dis 2004;189:1452-1465.

Address correspondence to: H.G. Sprenger, M.D. University Medical Center Groningen Dept. of Internal Medicine AA41 Hanzeplein 1

9713 GZ Groningen

The Netherlands

E-mail: h.g.sprenger@int.umcg.nl 